\title{
Coupling of Flame Geometry and Combustion Instabilities Based on Kilohertz Formaldehyde PLIF Measurements
}

\author{
Patton M. Allison, Yuntao Chen, Matthias Ihme, and James F. Driscoll \\ Department of Aerospace Engineering, University of Michigan, Ann Arbor, MI 48109 \\ Department of Mechanical Engineering, Stanford University, Stanford, CA 94305
}

Colloquium: IC Engine and Gas Turbine Combustion;

Alternate: Turbulent Flames

Total Length by Method 1: 6220

Main Text: 3895

Nomenclature: 0

Equations: 55

References: 420

Figure 1: 207

Figure 2: 144

Figure 3: 174

Figure 4: 285

Figure 5: 137

Figure 6: 179

Figure 7: 311

Figure 8: 283

Table 1: 130

If needed, color reproduction charges will be paid. 


\begin{abstract}
In the past, flame geometry was found to play an important role in determining when strong pressure fluctuations associated with a combustion instability occur in a gas turbine model combustor. The goals of this study were to use flame surface area as a more accurate way to quantify the flame shape and the heat release rate, in lieu of chemiluminescence; to accurately resolve both the spatial structure and the time history of the heat release fluctuations, and to show that kilohertz formaldehyde planar laser-induced fluorescence provides a new way to achieve these goals. The dual-swirl burner, developed at DLR Stuttgart by W. Meier et al., was operated using dimethyl ether (DME) to study fluctuations in the flame surface area, flame brush, flame length, and heat release rate. To understand the instability, accurate measurements are needed of the correlation between heat release rate fluctuations and pressure fluctuations. Thus heat release rate must be recorded as a function of time and space. However conventional chemiluminescence offers only a line-of-sight measurement. High-speed $\mathrm{CH}_{2} \mathrm{O}$ PLIF was applied to study the motion of flame surfaces in response to the pressure oscillations. Results show that the flame surface density fluctuated at the acoustic frequency (typically $320 \mathrm{~Hz}$ ). The frequency of the combustion instability was found to increase as either the gas velocity or the flame speed was increased. These trends are consistent with previous $n-\tau$ theories that model the convective time delay. Strong pressure oscillations occur for conditions when the flame is nearly flat; the instability disappears when the flame becomes Vshaped. The explanation is that all points on a flatter flame have the same convective time delay but this is not true for the V-flame geometry.
\end{abstract}

Keywords: instability, flame surface density, dimethyl ether, formaldehyde PLIF, high-speed, gas turbine combustor

\title{
I. Introduction
}

Swirl flames are known to experience combustion instabilities when the heat released by combustion oscillates in phase with pressure oscillations, such that the Rayleigh criterion is met [1]. However, swirl creates a complicated flow pattern that can alter the flame shape and the strength of the heat release rate, affecting the phase difference between pressure and heat release fluctuations. Simple models [2] show that differential equations can be used to predict the frequency $(f)$ of the pressure oscillation, which depends on the natural frequency $\left(f_{0}\right)$ of the acoustic resonator. However, these models show that the observed frequency may differ from $f_{0}$ due to the convective time delay $\left(\tau_{c}\right)$ between oscillations in the inlet gas velocity and the heat release that occurs downstream. The time delay can depend on flame shape, flame location (liftoff height), and flame length.

A well-documented, canonical dual-swirl burner that undergoes a natural combustion instability at a frequency in the range between 250 to $480 \mathrm{~Hz}$ was studied. It is an axisymmetric swirler-design and exhibits the fundamental physics associated with gas turbine flames; it contains two swirling air streams which surround an annular fuel stream. Giezendanner et al. [3], Weigand et al. [4], and Steinberg et al. [5,6] and have made extensive measurements of the flow field dynamics of this gas turbine model combustor (GTMC) for a few methane-fueled conditions. They showed that the vortex core precesses at a frequency close to $515 \mathrm{~Hz}$ while the combustion instability occurs at $305 \mathrm{~Hz}$. They also showed that the vortex core undergoes periodic expansion/contraction at the acoustic frequency of $305 \mathrm{~Hz}$, and that this vortex motion is coupled with the heat release oscillations. In the present work, the burner was operated on dimethyl ether (DME) for two fueling conditions, which represent cases in which the instability is present and resonating, or absent and non-resonating. As well, the GTMC 
was operated at various air mass flow rates to investigate the presence of a convective-acoustic instability. There have been several previous studies of combustion instabilities, but the temporal structure and the role of flame geometry has not been thoroughly investigated with experiments in confined turbulent flames. The spatial distribution of the flame surface can be determined from 2D planar images of formaldehyde PLIF. In addition, by performing PLIF measurements at high-speed, the temporal evolution of the flame surface can be investigated in relation to thermoacoustic oscillations and turbulent flowfield structures.

The new aspects of the present work are the following. High-speed formaldehyde PLIF was conducted at $4 \mathrm{kHz}$ to investigate the time-resolved motions of the flame surface relative to the fluctuating pressure field at reacting conditions. The flame surface densities for resonant and non-resonant operating conditions are compared to understand the importance of the spatial distribution of flame surface area. Flame geometry and shape have a major effect on the presence and strength of the instabilities. This paper reports that there are significant differences between the spatial and temporal flame shape for resonant and non-resonant flames. Flame geometry can be used as an important qualitative metric in the testing and design of gas turbine combustors to achieve instability-free operating conditions. It is also shown that the flame geometry can be more accurately determined from high-speed PLIF measurements than from conventional chemiluminescence alone.

While chemiluminescence images can be taken at high-speed, the collected data represents line-of-sight integrated information about the flame. Spatial asymmetries will be averaged out due to this integration and time-resolved motions will appear to be symmetric. Most importantly though, is the ability to distinguish differences in increased heat release. The local heat release can be altered by fluctuations in flame surface area or changes to the local reaction rate [7]. Chemiluminescence sequences cannot distinguish between these two causes and can only report the total effect of increased heat release. However, high-speed PLIF measurements can capture the independent effect of changes in flame surface area.

Species profiles predicted by CHEMKIN in laminar premixed DME-air flames are similar to those of propane-air [8], but DME produces significant formaldehyde concentrations. Compared to methane flames, the concentration in DME flames can be 10 to 100 times greater depending on the state of mixing. This characteristic of DME is useful in high-speed PLIF because low pulse energies, typical of high-speed laser systems, make the measurement difficult in other fuels. Gabet et al. [9] concluded that DME breaks down into formaldehyde in the cool flame region of 900-1200 K. It can be seen that DME marks the heated fuel region and that the steepest formaldehyde gradient consistently occurs near the peak of the $\mathrm{CH}$ radical production, which is a good marker of the heat release layer. While measurements of CH-PLIF would certainly provide an ideal flame front marker, it is not a readily feasible measurement at high repetition rates due to the energy limitations of most current laser systems. Formaldehyde provides an ideal flame front marker for thin preheat zone structures, in lieu of broad hot product $\mathrm{OH}$ marking, for high-speed tracking. 
Several types of combustion instabilities can occur simultaneously in swirl-stabilized flames [10, 11]. Convectiveacoustic modes $[12,13]$, are due to the coupling between the flow velocity and a convected entropy wave. Our previous measurements [14] and work by Steinberg et al. [6] showed that the GTMC is a Helmholtz resonator that undergoes equivalence ratio oscillations; the frequency of the instability depends on the flow velocity and the flame speed, thus convective effects are important.

The primary goal of this study was to use the flame surface area as a more accurate way to quantify the flame shape and the heat release rate, in comparison to chemiluminescence measurements. The second objective was to apply high-speed PLIF diagnostics to accurately resolve both the spatial structure and the time history of the heat release rate.

\section{Experimental Apparatus}

The gas turbine model combustor [4], shown in Figure 1, is used to study the combustion instability. The experimental setup was replicated from DLR Stuttgart, but performed at the University of Michigan. The injector consists of a central air nozzle with outer diameter (OD) of $15 \mathrm{~mm}$, an annular fuel nozzle, and a co-annular air nozzle (ID = 17mm, OD = $25 \mathrm{~mm}$ ). Both air nozzles supply swirling air at atmospheric pressure and temperature from a common plenum. The exit plane of the central air nozzle and fuel nozzle lies $4.5 \mathrm{~mm}$ below that of the outer air. This recession causes partial-premixing of the reactants in the region upstream of the flame [14]. The combustion chamber has a square cross section of $85 \mathrm{~mm}$ in width and $110 \mathrm{~mm}$ in height. The exit of the combustion chamber is an exhaust tube with a diameter of $40 \mathrm{~mm}$ and a height of $50 \mathrm{~mm}$. Mass flow rates were controlled by choked orifices that have accuracies of $\pm 2.25 \%$. The air mass flow rate for both cases compared was $282 \mathrm{~g} / \mathrm{min}$ and the global equivalence ratio for the non-resonant and resonant cases were 0.75 and 1.20 , respectively. The study of convective-acoustic effects was performed over a range of air mass flow rates $(170-355 \mathrm{~g} / \mathrm{min})$ at a global equivalence ratio of 1.0 for several fuels.

In this study, the $4_{0}^{1}$ transition in formaldehyde is excited using a frequency-tripled diode pumped Nd:YAG Quantronix Hawk HP laser at $355 \mathrm{~nm}$. At $4 \mathrm{kHz}$, the laser energy per pulse was $9.7 \mathrm{~mJ}$ and the sheet height was $25 \mathrm{~mm}$. Fluorescence was recorded with LaVision HS-IRO intensifier, with a gate time of $200 \mathrm{~ns}$, a Phantom v711 camera, and a 105 mm Nikon micro-Nikkor f/2.8 lens. Spatial resolution was $76 \mu \mathrm{m}$ per pixel. A field of view of $80 \mathrm{~mm}$ by $20 \mathrm{~mm}$, which covers the width of the burner and the entire height of the flame, was observed. A GG-385 filter removed laser interference below $385 \mathrm{~nm}$. After background corrections and filtering, the signal to noise ratios varied between 6.5-11.2.

A PCB piezoelectric transducer (112A04) was mounted in the side wall of the plenum. The plenum has a diameter of 80 $\mathrm{mm}$ and a length of $72 \mathrm{~mm}$. For a given operating condition, pressure measurements are taken at a set burner reference 
temperature. The frequency and amplitude in the plenum was previously shown to be not significantly different from that in the combustion chamber, with the exception of a phase shift [16]. The reported frequencies are accurate to $\pm 0.5 \%$.

\section{Results and Discussion}

\section{A. Global Flame Geometry and Presence of Combustion Instabilities}

Figure 2 shows that there are noticeable differences in the flame shapes between the resonating case and the nonresonating case, as determined by time-averaged chemiluminescence. The non-resonating flame has an axially extended Vshape profile. Flat flames are thermally compact and concentrate heat release in a smaller volume than the V-flame. It was previously determined from LDV velocity measurements that the flame shape is set by the distribution of air flow rate through the two swirlers [17]. Resonating flames exhibited a larger measured radial velocity at the exit of the nozzle due to higher flow through the outer swirler. Higher radial velocities are essential to the establishment of flat flames. When the flame was non-resonant, low radial flow rates were observed and the flame had a V-shape. Recent LES-computations of the stable operating point [18] showed that the shape of the flame is controlled by the vortex-breakdown, flow-field separation at the outer swirler and the mass-flow split.

The spatial location of the flame alters the convective time scales and thus determines the degree to which the instability is excited. The convective-acoustic mode is controlled by two timescales, a convective timescale for perturbations to reach the flame and an acoustic time scale for perturbations to return to the nozzle. The instability frequency is inverse to the sum of these scales. For relatively flat flames, this convective time scale is uniform across the flame surface. However, for V-shaped flames, the distance to the flame varies over the length of the surface, and thus the time scale also varies, as seen by the arrows in Figure 2. Thus in flat flames, if the phase difference is appropriate, the entire flame contributes to the amplification. However, in V-shaped flames, only a portion of the flame surface may have the correct phase difference to aid in amplification, and the resonance may not occur.

Figure 3 provides evidence that a convective-acoustic mechanism is present because the frequency of the instability is found to scale with the gas velocity. This mechanism often is associated with equivalence ratio oscillations. Inhomogeneities in fuel-air ratio can create packets of fluid that are convected and lead to unsteady heat release. Pressure fluctuations that are reflected back to the injector provide the feedback that triggers the formation of the next packet. The frequency of the feedback is set by the characteristic time of the cycle for packets to be convected to the flame and then have pressure waves reflected back. This characteristic time is reduced if convection speeds are increased, thus increasing the frequency of the instability, as seen in Figure 3. 


\section{B. Flame Surface Density and Surface Area from kHz PLIF}

The flame surface density (FSD) here is used as an indicator of the local volumetric heat release rate $\left(q^{\prime}\right)$; this was done previously by Steinberg et al. [6], and the two quantities were shown to be correlated in the DNS work of Tap et al. [19]. Also, the subgrid reaction rate $\left(\omega_{\mathrm{sg}}\right)$, which is proportional to heat release rate, has been modeled in [20, 21] to be proportional to the reactant density, laminar flame speed, and FSD. To mark the flame surface, the steep gradient on the flame side of the formaldehyde region is used, since it corresponds to the maximum production of $\mathrm{CH}$ radicals in the reaction zone, as predicted by CHEMKIN simulations of a freely propagating DME flame.

The flame surface was identified using edge marking and by defining a gradient threshold. The high gradient edge where the flame exists is easily distinguished from low PLIF signal gradients that result from fuel decomposition. Figure 4a is a typical PLIF image that is then binarized to eliminate background noise. A Canny edge tracking method marks the detected edges that are seen in Figure 4b. Next, the gradient is calculated along this edge using a central difference method on a $4 x 4$ stencil at each edge marker. Gradients that exceed a threshold are defined to be flame surfaces. The remaining edge markers in Fig. 4c satisfy the threshold of 10 counts/pixel, while the missing pixels denote the edges that did not pass the threshold requirement. Those below the threshold are considered markers of fuel decomposition into formaldehyde or noise in the signal causing false edges.

The flame surface density is the average area of flame surface that lies within a 3-D volume in space. Given that the formaldehyde image measurements are 2-D in nature, the FSD can be estimated by:

$$
F S D_{x y}=\frac{\delta L_{f}}{\delta A_{x y}}
$$

where $\delta L_{f}$ is total flame length that lies inside the interrogation box area $\delta A_{x y}$ in 4000 images. Each box is 0.76 x $0.76 \mathrm{~mm}$, as given by a $10 \times 10$ pixel box. Figure 5 depicts the measured FSD for the resonating and non-resonating cases. The width of the contours indicates the width of the turbulent flame brush. While there may exist some out of plane motion, the FSD may be affected. However, the primary concern is that any flame surface captured in the plane will contribute to the fluctuating heat release.

Compared to the chemiluminescence in Figure 2, the structure of both cases appears different. This is due primarily to differences in line-of-sight versus in-plane imaging. With the in-plane imaging, the spatial localization is better defined and a clearer visual of the radial location of heat release is observed. For both cases, flame surface density is low on the centerline. The non-resonating case has more FSD at radially-inward locations, while for the resonating case the FSD is distributed radially further out. In both cases, the FSD is confined within two lobes that are symmetric around the centerline. For the 
non-resonant case, each lobe is seen to be composed of two separate zones. Previous PIV measurements [6] showed that a shear layer exists at the location between these two zones. This layer separates the central and outer recirculation zones and may explain why each FSD lobe is divided into two regions in Fig. 5a.

Figure 5 shows that the highest probability of flame occurrence is at the outer edges of the brush. The non-resonating case displays an initial rise in flame surface marking the inner zone of each lobe, followed by a secondary rise marking the outer zone of the lobes. The resonating case shows that the maximum wrinkling occurs at a larger radial distance. With higher turbulence intensities, it is expected that the flame would become more wrinkled and the total flame surface area would increase. This is not apparently clear from measurements of flame length, which give approximately the same average inplane length for both cases, as seen in Table 1. In fact, the total integrated FSD for each case results in roughly the same degree of flame surface. However a more important metric would be the 3D surface area of the flame, which, assuming that the flame is axisymmetric, can be estimated by the convolution of the flame surface length with its radial position. This results in a radially-weighted surface area parameter, such that wider flames will have a larger area. This metric dictates that flame surface which is distributed further from the centerline contributes more to the total surface area of the flame. The flame area per unit length parameter $\Psi$ is defined to be:

$$
\Psi=\int_{-\infty}^{+\infty} r \cdot F S D d h
$$

where $\Psi$ is proportional to the flame area per unit distance $(\delta r)$ in the radial direction. The radial profiles of $\Psi$ that are plotted in Fig. 6 indicate the radial location where flame surface density is maximum. This is consistent with the FSD contours of Figure 5, but the $\Psi$ parameter is weighted by the radial coordinate (r) which appears in Eq. 2 . Figure 6 suggests that a sufficiently large value of $\Psi$ (surface area per unit length in the radial direction) coincides with the presence of a combustion instability, which globally means that the flame brush is flat and thin. The non-resonating flame has a smaller profile of $\Psi$ because of its V-shape. By having flame surface occur at large radial locations, as in flat flames, the convoluted flame surface area is greater, which is correlated with larger heat release. Coupled with the instability, fluctuations in flame surface at a large radial distance also result in larger heat release fluctuations. If the appropriate phase difference occurs between the heat release and pressure fluctuations, large surface area oscillations at large radial distances can strengthen and amplify the instability even further. For resonating flames, the average area across all images is $369 \pm 128 \mathrm{~cm}^{2}$ and for nonresonating flames, the average is $305 \pm 66 \mathrm{~cm}^{2}$. The resonating flame displays a larger area by $20 \%$ and the range of fluctuations is larger. 


\section{Time Variations of the Flame Surface}

If the flame surface density oscillation (i.e., local heat release rate) is a direct cause of the combustion instability, then it would be expected that FSD should oscillate at nearly the same frequency as the pressure oscillations. To assess this idea the kHz PLIF data was interrogated and the results are listed in Table 1. For the resonating case, the FSD oscillates at $320 \mathrm{~Hz}$, as seen from the spectrum of FSD in Figure $7 \mathrm{~b}$. This result dictates that the flame surface area is modulated at the observed pressure fluctuation frequency and that the heat release will also oscillate at the acoustic frequency. Similar behavior was observed from complementary high-speed OH-PLIF results by Steinberg et al. [5]. For the non-resonant case, the pressure fluctuations are negligible, however the FSD fluctuates at $10 \mathrm{~Hz}$ as seen in the spectrum of Figure 7a. This can be described as a slow breathing mode which scales with the radial velocity fluctuations and the diameter of the GTMC.

From the high-speed videos of the motion of the formaldehyde scalar field, it is apparent that there is a lateral variation in time of increased FSD from one lobe to another. If each lobe is considered as an independent region of interest, the time evolution can be considered, which will isolate asymmetric behavior in the flame surface. This is lost when considering the combined motions of both lobes within the entire field of view. For resonant flames, the total integrated FSD in each lobe independently oscillates at the acoustic frequency. However, in the non-resonant case, there is a strong oscillation observed at $475 \mathrm{~Hz}$. Although, not directly measured in this study, measurements taken at DLR Stuttgart by Boxx et al. [22] have contributed motions near this frequency to be caused by the rotation of the PVC.

The relative time-resolved behavior of each lobe can be considered by evaluating the ratio of the total flame surface in each lobe. The relative lobe motion captures the in-plane change in FSD across the centerline as affected by the PVC motion. For the non-resonant flame, the signal from one lobe to the other fluctuates at $475 \mathrm{~Hz}$, corresponding to the PVC, as seen in Figure 8a. However, in the resonating flame, the ratio of the signal oscillates at $525 \mathrm{~Hz}$, given in Figure $8 \mathrm{~b}$. The other large peaks correspond to the acoustic frequency $(320 \mathrm{~Hz})$ and the difference between the PVC rate $(525 \mathrm{~Hz})$ and the acoustic frequency $(320 \mathrm{~Hz})$, which is $(205 \mathrm{~Hz})$. These peaks were also observed by Steinberg et al. [6] from high-speed PIV. Again, it is hypothesized that this oscillation corresponds to the rotation of the PVC through the plane of the laser sheet. In contrast

to the total FSD spectra in Figure 7, the relative lobe motion spectra exhibit strong peaks at the hypothesized PVC rotation frequency. From the time history of the signal in each lobe, it was observed that there is a phase shift between the temporal behaviors of each lobe, such that the amount of flame surface present in a given lobe may relatively lead/lag behind the other. There was a phase shift of $127^{\circ}$ in non-resonant flames and a shorter phase shift of $89^{\circ}$ in resonant flames corresponding to the relative faster rotation of the PVC. 
The time-resolved behaviors observed in this case were captured with the ability to better identify spatial variations in flame with the use of high-speed PLIF. For both cases, large radial variations in FSD were observed and there appears to be influences of the PVC on the motion of the flame surface. This behavior is asymmetric due to the helical structure of the PVC and would not be captured in line-of-sight chemiluminescence images.

\section{Conclusions}

A combustion instability was studied in a gas turbine model combustor by varying the air velocity, the flame speed and the flame shape. Dimethyl ether was used to maximize the signal from the kilohertz PLIF formaldehyde diagnostics. The pressure spectrum for the acoustically excited mode has a single peak and the frequency was found to increase nearly linearly as the air velocity is increased. This indicates that the instability is convective-acoustic in nature. The frequency also increases with flame speed, based on comparisons to our data for faster burning fuels.

Models of such instabilities show that the convection time scale determines the amplitude and frequency of the instability. It was observed that the flame shape plays an important role; flat flames exhibit a strong instability whereas V-

shaped flames are typically quiet. For flat flames, the entire flame has the same convective time scale. This convection time scale varies along a V-flame, so only a small segment of the flame can amplify the pressure oscillations. The flame geometry in this GTMC is controlled by mixing and the upstream flow pattern; specifically the amount of radial velocity imparted by the distribution of flow through each swirler.

It is shown that the flame geometry, including its spatial and temporal variations, can be more accurately determined using kHz PLIF than by conventional chemiluminescence. Spatial variations of flame surface density (and heat release rate) in resonating flames differed from that of non-resonating flames. The resonating case has a larger flame surface area and the peak FSD was located at larger radial distances than for the non-resonating case. When the instability is present, the magnitude of area fluctuations was much greater, indicating large scale surface creation and destruction. The combination of large surface area and large area fluctuations leads to increased amplification of the instability.

The strength of high-speed PLIF was observed in the analysis of the time evolution of the flame surface structure with the spectrum of flame surface area oscillations. Surface area and thus heat release rate were seen to oscillate at the same $320 \mathrm{~Hz}$ frequency as pressure fluctuations. This coupling of pressure and heat release oscillations leads to amplification of the instability based on the Rayleigh criterion. However, the flame surface also exhibited asymmetric motions at $525 \mathrm{~Hz}$ associated with the presence of the PVC. For non-resonating flames, oscillations in flame surface density also occur at a lowfrequency $(10 \mathrm{~Hz})$ due to a breathing mode and at the PVC rotation rate $(475 \mathrm{~Hz})$. 


\section{Acknowledgments}

This research was funded by ONR under grant N00014-10-10561 and by the DOE-UTSR program under grant FE0007060. The authors thank Dr. Wolfgang Meier of DLR Stuttgart for permission to use the GTMC design.

\section{References}

[1] J.W.S. Rayleigh and R. Lindsay, The Theory of Sound: Vol. 2, Dover Publications, 1945, p. 230.

[2] J. P. Hathout, M. Fleifil, A. M. Annaswamy, A. F. Ghoniem, J. Propulsion and Power 18 (2) (2002) 390-399.

[3] R. Giezendanner, P. Weigand, X.R. Duan, W. Meier, U. Meier, M. Aigner, and B. Lehmann, J. Eng. Gas Turb. Power 127 (2005) 492-496.

[4] P. Weigand, W. Meier, X.R. Duan, W. Stricker, and M. Aigner, Combust. Flame 144 (2006) $205-224$.

[5] A.M. Steinberg, I. Boxx, M. Stöhr, C.D. Carter, and W. Meier, Combust. Flame 157 (2010) 2250-2666.

[6] A.M. Steinberg, I. Boxx, M. Stöhr, W. Meier, and C.D. Carter, AIAA J., 50 (4) (2012) $952-967$.

[7] J.G. Lee and D.A. Santavicca, J. Propulsion and Power 19 (5) (2003) 735 - 750.

[8] C.A. Daly and J.M. Simmie. Combust. Flame 125 (4) (2001) 1329-1340.

[9] K. Gabet, H. Shen, R. Patton, F. Fuest, and J. Sutton, Proc. Combust Inst. 34 (2013) 1447-1454.

[10] S. Ducruix, T. Schuller, D. Durox, and S. Candel, J. Propulsion and Power 19 (5) (2003) 722 - 734.

[11] B.T. Zinn and T. Lieuwen, in: T. Lieuwen and V. Yang (Eds.), Combustion Instabilities in Gas Turbine Engines, AIAA, 2005, p. $9-13$.

[12] K.H. Yu, A. Trouvé, and J.W. Daily, J. Fluid Mech 232 (1991) 47 - 72.

[13] T. Lieuwen, H. Torres, C. Johnson, and B.T. Zinn, J. Eng. Gas Turb. Power 123 (2001) 182-189.

[14] P.M. Allison, M. Ihme, and J.F. Driscoll, Proc. Combust Inst. 34 (2013) 3145-3153.

[15] W. Meier, X.R. Duan, and P. Weigand, Proc. Combust Inst. 30 (2005) 835-842.

[16] R. Sadanandan, M. Stöhr, and W. Meier, Combustion, Explosion, and Shock Waves 45 (2009) 518-529.

[17] P. M. Allison, D.A. Rosenberg, and J.F. Driscoll, Combustion instability interaction with fuel-air mixing in a partiallypremixed gas turbine model combustor: Syngas and hydrocarbon fuel comparisons, $8^{\text {th }}$ Mediterranean Combustion Symposium, International Centre for Heat and Mass Transfer (2013).

[18] Y. C. See and M. Ihme, Large eddy simulation of a partially-premixed gas turbine model combustor. Proc. Combust Inst. 35 (2014), accepted for presentation.

[19] F.A. Tap, R. Hilbert, D. Thevenin, and D. Veynante, Combust. Theory Modelling 8 (2004) 165-193.

[20] M. Boger, D. Veynante, H. Boughanem, and A. Trouve, Proc. Combust. Institute 27 (1998) 917-925.

[21] M.P. Kirkpatrick, S.W. Armfield, A.R. Masri, S.S. Ibrahim, Flow, Turb. Combust. 70 (2003) 1-19.

[22] I. Boxx, M. Stöhr, C. Carter, W. Meier, Combust. Flame 157 (2010) 1510-1525. 
Table 1. Summary of temporal flame surface responses in resonant and non-resonant DME flames.

\begin{tabular}{lcc}
\hline \multicolumn{1}{c}{ Fuel Type } & DME & DME \\
\multicolumn{1}{c}{ Air [g/min] } & 282 & 282 \\
Equivalence Ratio, $\phi$ & 0.75 & 1.2 \\
\hline Instability Present & No & Yes \\
Acoustic Frequency [Hz] & - & 320 \\
FSD Frequency [Hz] & 10 & 320 \\
Individual Lobe Frequency [Hz] & 475 & 320 \\
Lobe Ratio Frequency [Hz] & 475 & 525 \\
Lobe Phase Shift [deg] & 127 & 89 \\
Flame Surface Area Mean [ $\left.\mathrm{cm}^{2}\right]$ & 305 & 369 \\
Flame Surface Area RMS [ $\mathrm{cm}^{2}$ ] & 65.6 & 128 \\
Flame Length Mean [mm] & 143 & 135 \\
Flame Length RMS [mm] & 28.2 & 48 \\
\hline \hline
\end{tabular}

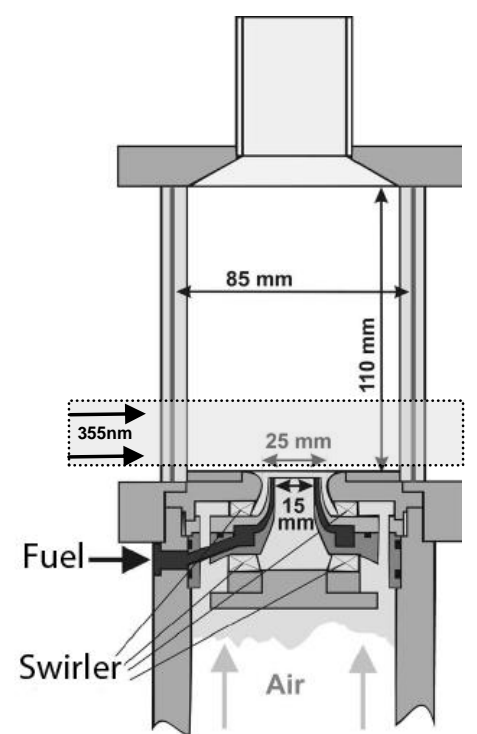

Figure 1. The Gas Turbine Model Combustor designed by Meier et al [4]. Formaldehyde excited by $355 \mathrm{~nm}$ laser sheet aligned with the burner centerline. 


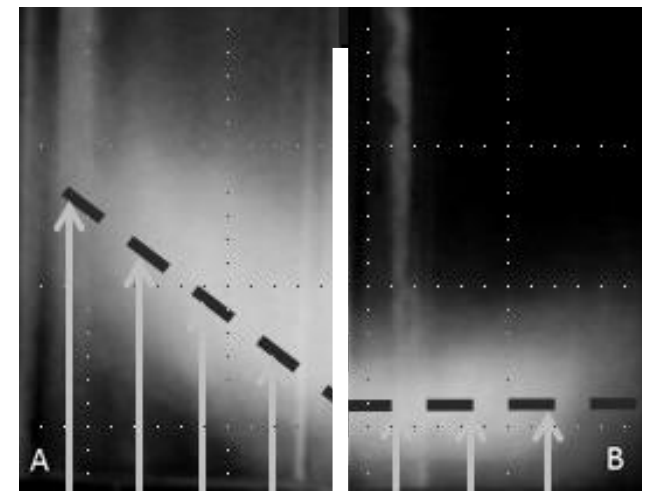

Figure 2. Time-averą ed

chemiluminescence of $\mathbf{V}$-shaped, nonresonant flames (a) and flat, resonating flames (b).

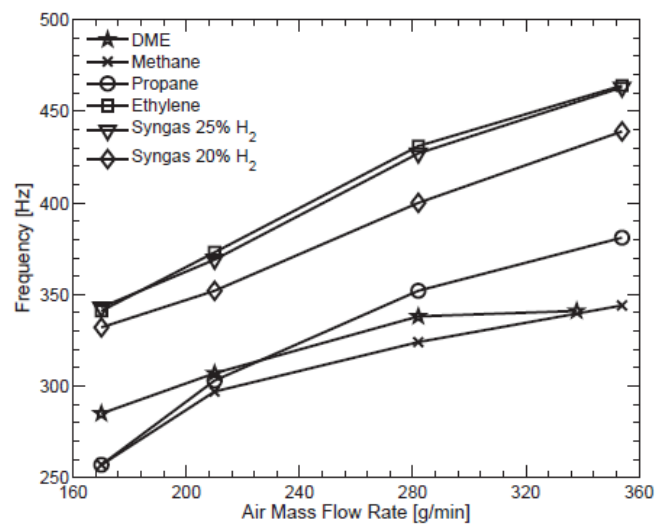

Figure 3. Frequency of the instability of the present DME flames (stars) compared to other fuels [13]. Frequency increases with gas velocity and air mass flow rate indicating that a convective-acoustic mechanism is present. $\varphi=\mathbf{1 . 0}$. 


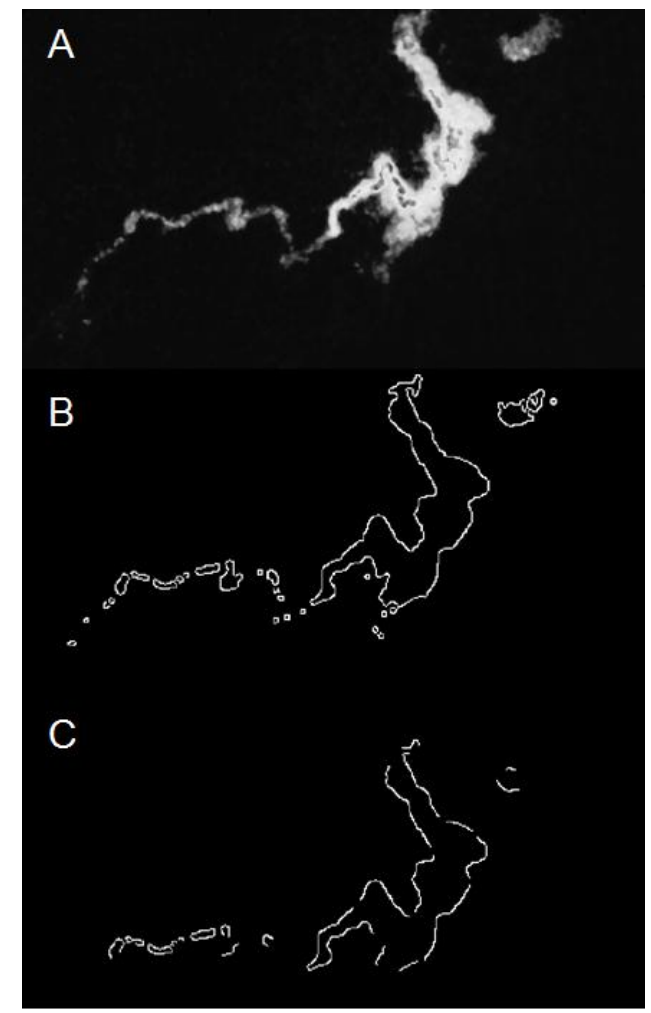

Figure 4. Detection method of flame surface segments from formaldehyde PLIF images. Raw image (a) and all detected edges (b). In (c), the remaining white edges are segments marked as flame surface and the missing edges have been neglected by the gradient threshold.
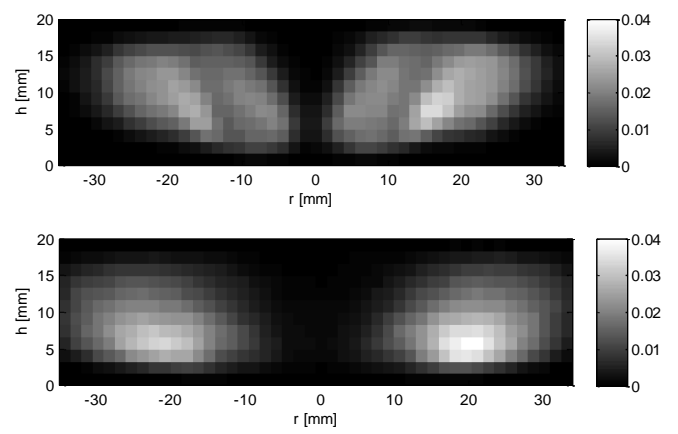

Figure 5. Mean flame surface density $\left(\mathrm{mm}^{-1}\right)$ for non-resonant (top) and resonant (bottom) DME flames. 


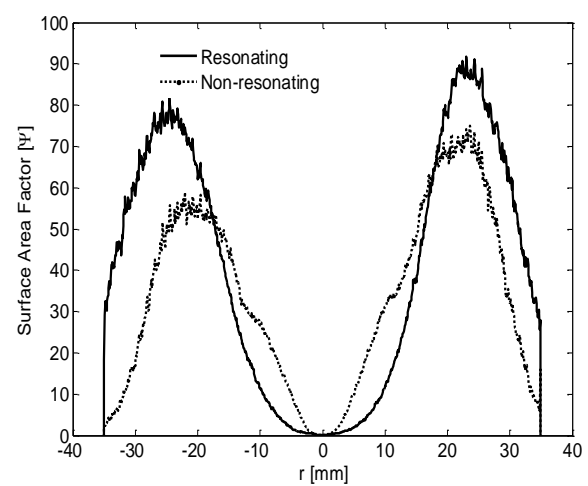

Figure 6. Radial distributions of flame surface area parameter $\Psi$ (defined by Eq. 2 ) depicting the distribution of flame surface transversely across the burner. 

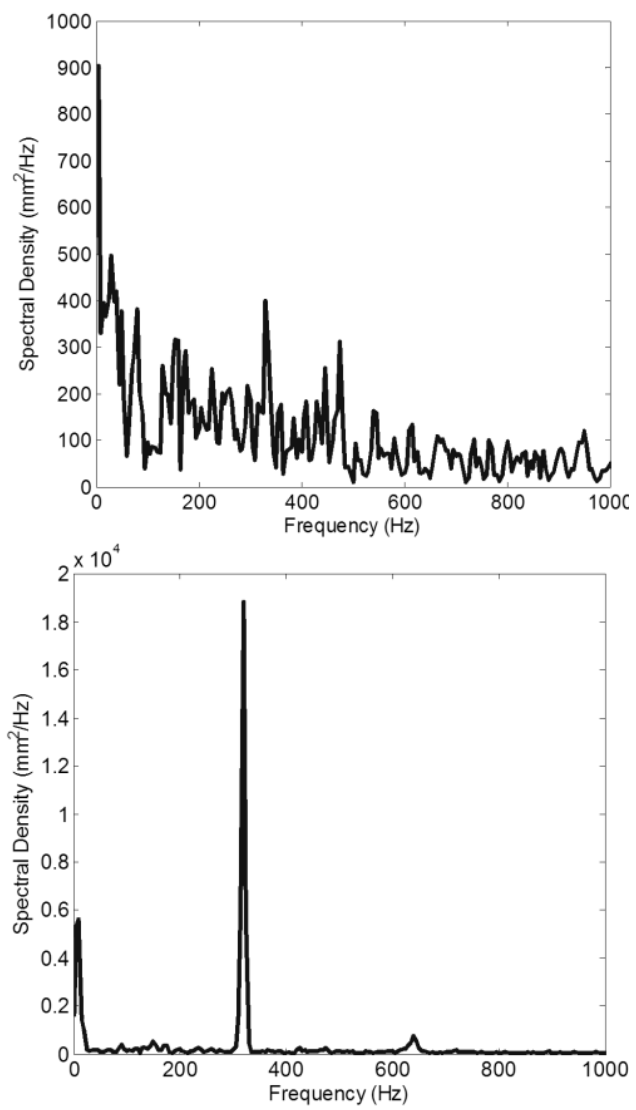

Figure 7. Spectra of flame surface density from $\mathrm{kHz}$ PLIF images. (a) Nonresonating flame showing a peak at $10 \mathrm{~Hz}$. (b) Resonating flame, showing much larger peaks at $10 \mathrm{~Hz}$ and $320 \mathrm{~Hz}$. The $320 \mathrm{~Hz}$ peak corresponds to the frequency of pressure oscillations and $10 \mathrm{~Hz}$ is an asymmetric breathing mode. 

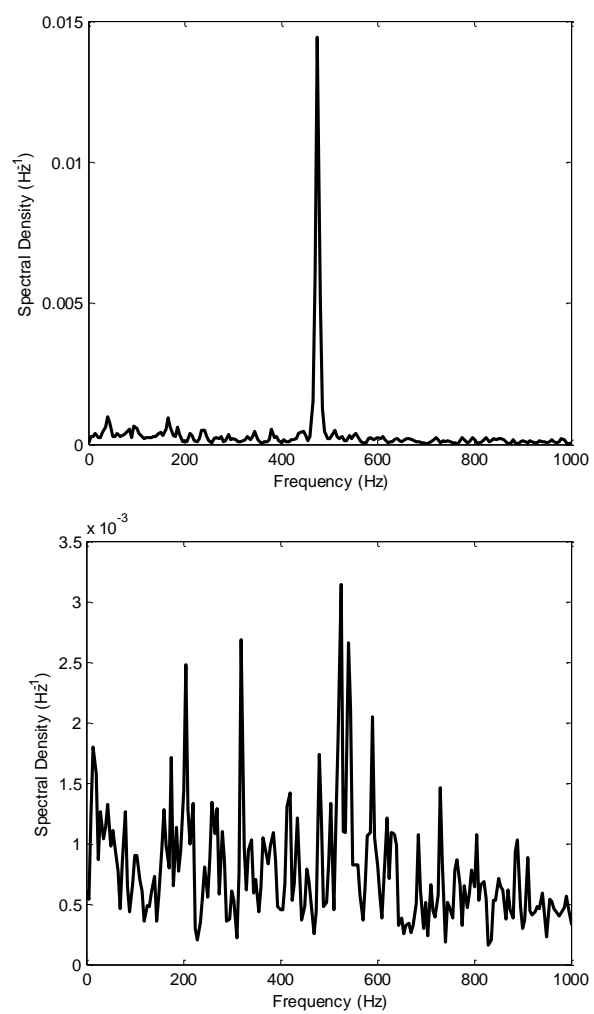

Figure 8. Spectra of the ratio of flame surface length fluctuations in each lobe from $\mathrm{kHz}$ PLIF images. (a) Nonresonating flame showing a peak at 475 $\mathrm{Hz}$ corresponding to the asymmetric motion of the PVC across the centerline of the burner. (b) Resonating flame, showing motion of the PVC at $525 \mathrm{~Hz}$. 
Figure Captions:

Figure 1. The Gas Turbine Model Combustor designed by Meier et al [4]. Formaldehyde excited by 355nm laser sheet aligned with the burner centerline.

Figure 2. Time-averaged chemiluminescence of $\mathrm{V}$-shaped, non-resonant flames (a) and flat, resonating flames (b).

Figure 3. Frequency of the instability of the present DME flames (stars) compared to other fuels [13]. Frequency increases with gas velocity and air mass flow rate indicating that a convective-acoustic mechanism is present. $\varphi=1.0$.

Figure 4. Detection method of flame surface segments from formaldehyde PLIF images. Raw image (a) and all detected edges (b). In (c), the remaining white edges are segments marked as flame surface and the missing edges have been neglected by the gradient threshold.

Figure 5. Mean flame surface density $\left(\mathrm{mm}^{-1}\right)$ for non-resonant (top) and resonant (bottom) DME flames.

Figure 6. Radial distributions of flame surface area parameter $\Psi$ (defined by Eq. 2) depicting the distribution of flame surface transversely across the burner.

Figure 7. Spectra of flame surface density from kHz PLIF images. (a) Non-resonating flame showing a peak at $10 \mathrm{~Hz}$. (b) Resonating flame, showing much larger peaks at $10 \mathrm{~Hz}$ and $320 \mathrm{~Hz}$. The $320 \mathrm{~Hz}$ peak corresponds to the frequency of pressure oscillations and $10 \mathrm{~Hz}$ is an asymmetric breathing mode.

Figure 8. Spectra of the ratio of flame surface length fluctuations in each lobe from kHz PLIF images. (a) Nonresonating flame showing a peak at $475 \mathrm{~Hz}$ corresponding to the asymmetric motion of the PVC across the centerline of the burner. (b) Resonating flame, showing motion of the PVC at $525 \mathrm{~Hz}$.

Table 1. Summary of temporal flame surface responses in resonant and non-resonant DME flames. 